\title{
Reducing Energy Usage in Drive Storage Clusters through Intelligent Allocation of Incoming Commands
}

\author{
E. Smart ${ }^{\mathrm{a}, *}$, D. Brown ${ }^{\mathrm{a}}$, K. Toumi Borges ${ }^{\mathrm{b}}$, N. Granger-Brown ${ }^{\mathrm{b}}$ \\ ${ }^{a}$ Institute of Industrial Research, 36-40 Middle Street, University of Portsmouth, \\ Portsmouth, Hampshire, PO5 4BP, United Kingdom. \\ ${ }^{b}$ Seagate Technology, Langstone Road, Havant, Hampshire, PO9 1SA, United Kingdom
}

\begin{abstract}
Although significant research has been undertaken to reduce high level energy consumption in a data centre, there has been very little focus on reducing storage drive energy consumption via the intelligent allocation of workload commands at the file system level. This paper presents a method for optimising drive energy consumption within a custom built storage cluster containing multiple drives, using multi-objective goal attainment optimization. Significantly, the model developed was based on actual power consumption values (from current/voltage sensors on the drives themselves), which is rare in this field.
\end{abstract}

The results showed that command energy savings of up to $87 \%$ (17\% overall energy) could be made by optimising the allocation of incoming commands for execution to drives within a storage cluster for different workloads. More significantly, the transparency of the method meant that it showed exactly

\footnotetext{
*Corresponding author. Tel: +44 (0) 239284 4448, Fax: +44 (0) 2392845192

Email addresses: edward.smart@port.ac.uk (E. Smart), david.j.brown@port.ac.uk (D. Brown), kais.toumi@seagate.com (K. Toumi Borges), nick.grangerbrown@seagate.com (N. Granger-Brown)
} 
how such savings could be made and on which drives. It also highlighted that whilst it is well known that solid state drives use less energy than traditional hard disk drives, the difference is not consistent for different sizes of data transfers. It is far larger for small data transfers (less than or equal to $4 \mathrm{kB}$ ) and our algorithm utilised this.

Significantly, it highlights how much larger energy savings can be made through using the optimisation results to show which drives can be safely put into a low power state without affecting storage cluster performance. Keywords: Decision support systems, disk drives, data storage systems, pareto optimization, optimal scheduling

\section{1. Introduction}

2 Data centres are a key part of the world's economic infrastructure and 3 vary in size from server farms that support the likes of Google and Facebook 4 to the server rooms that support small-to-medium corporations. This heavy 5 reliance on data has made data centres one of the biggest consumers of elec6 tricity (estimated at 91 billion kilowatt hours in America alone in 2013) ${ }^{1}$. 7 However, according to the same report, as much as $50 \%$ of this is wasted. 8 As such, energy efficiency in data centres is a key topic for the research 9 community.

10 The topic of energy reduction within a data centre is well researched with 11 significant activity in the following areas; power usage within a disk drive, 12 reducing disk drive power states within a storage cluster and intelligent data 13 assignment. However, nearly all of this research focusses on high level data 14 flow within sets of storage clusters. 
Our research focuses on the problem from a new angle and analyses energy management at a much lower level. It looks at modifying the way in which read/write commands are managed within a storage system in order to minimise the energy used in this process. The algorithm proposed is intended to operate at a level between the operating system and the storage devices and would be embedded within the kernel. The algorithm would then take commands that come from the upper layers and redirect them to the most appropriate drives. To conceptually illustrate the algorithm in action, suppose that the file storage system is blank. If a given application wants to store data that would be rarely retrieved (read), the algorithm would redirect that data to drives with the least write energy costs. However, if the data was to be retrieved (read) often, it would be stored (written) on those drives with the lowest read energy costs (even if they had higher write energy costs than other drives). This would occur until all of the storage devices were full, thus creating a storage system with an overall low optimal energy consumption profile.

The following areas are out of scope of this piece of research; algorithm implementation, where it should sit within the storage system and also what happens to data once it has been initially written (e.g. should it be replicated onto other drives).

We propose the use of the multi-objective goal attainment algorithm to optimally assign individual commands to drives in order to achieve minimal command energy usage at the storage cluster level. In storage clusters, it is not unreasonable to see multiple copies of regularly accessed files stored on different drives (e.g. popular YouTube videos). This means that any energy 
40

management algorithm could access a file by reading different sections of it from different drives. To the best of the author's knowledge, there are no other methods that operate so deep within the file system at such a low level. For this reason, it was not meaningful to compare the proposed method with others in the literature review (see section 2).

A custom built storage cluster was fitted with two types of drive: solid state drive (SSD) and hard disk drive (HDD). Their energy usage was computed directly using current and voltage sensors to ensure accurate values. This is a significant advantage over previous research (e.g. Chou et al. ${ }^{2}$ ) as usually storage clusters are simulated numerically, which can lead to inaccuracies if drive background activity is not taken into consideration.

The paper is organised as follows. Section 2 contains the literature review. Section 3 describes the multi-objective goal attainment method. Section 4 explains the experimental setup. Section 5 describes the method. Section 6 details the results and section 7 lists the conclusions.

\section{Literature Review}

In this paper, we assess the available literature for energy saving methods, hardware and software based, for disk storage clusters as well as for optimisation methods.

\subsection{Storage Cluster Energy Saving}

The main methods used to perform energy saving focus on general dynamic power techniques and general performance enhancing techniques that additionally improve power usage. 
Modern drives have multiple power states such as 'active' (data transfer ready), 'idle' (waiting for data transfer) and 'standby' (some electronics turned off and disk platters stopped $)^{3}$. For more details on drive power states, see section 4.4. Significant power savings can be made by putting HDDs into standby mode but if a drive receives a new I/O request, it takes time and energy to spin up, which often has too much of an impact on performance in certain applications. The duty cycle rating is defined as the number of spin ups and spin downs a disk can withstand before the failure risk on spin up exceeds $50 \%$. For server based drives, this figure is much less than for laptop drives so energy saving measures should not spin up or spin down a drive indiscriminately ${ }^{4}$.

A basic approach is to use Traditional Power Management (TPM) methods, which are usually threshold based ${ }^{5}$. In such methods, drive power states are changed after a given threshold time $\tau$, which can be static or dynamic. This can be effective if the pattern of $\mathrm{I} / \mathrm{O}$ requests is known but for the more common unknown circumstance, it can be shown that by using competitive analysis to set $\tau$ to the break-even time, this policy uses at most twice the power of the most optimal offline policy.

Another proposed method is to use variable speed disk drives ${ }^{6}$. Rather than having 2 discrete states (idle and standby), this approach lets drives spin at different speeds so that at least a few will be at full speed to service requests. It has been shown that this is a good way to manage the balance between performance and energy usage.

A more common approach is to use Dynamic Power Management (DPM) techniques, which seek to maximise energy savings by distributing I/O re- 
quests over time and across all the disks in the array ${ }^{7}$. It achieves energy savings by first controlling the frequency of I/O requests to each disk so that the mean idle time is large whilst maximising the idle-time variance across all the disks and through time. Techniques include power aware caching and buffering in memory ${ }^{8}$, across disks $^{9}$, workload consolidation through data replication ${ }^{10}$, popular data concentration ${ }^{11}$ and diverting disk accesses by utilising data redundancy ${ }^{12}$.

Previous work has been undertaken to analyse the performance of drives and storage clusters. Härder et al. ${ }^{13}$ looked at the performance and power consumption of SSDs in a storage cluster and found significant differences in consumption. However, it is difficult to assess this as power consumption was not measured directly from the drives themselves and it was not stated whether the drives were performing maintenance actions at the time.

Attempts at energy reduction in storage systems are usually made at a higher system level. For example, improved methods of heat dissipation via different types of coolants or through different systems architecture. Our approach seeks to focus on the properties of the different drive types and the types of commands they are able to process more efficiently (with respect to energy) so that workload commands can be assigned optimally.

\subsection{Optimisation Methods}

To the best of the authors knowledge, there have been no attempts to apply optimisation methods to assign commands to drives. The basic problem is to assign $n$ commands to $m$ drives where $n$ is much larger than $m$ and both are natural numbers. This could be tackled by means of a semi-assignment algorithm. Drwal ${ }^{14}$ used this method to assign streams of requests to servers 
in order to minimise the worst case processing times although he offers no details as to how his algorithm improves the total time. Vasudevan ${ }^{15}$ uses a semi-assignment algorithm to optimally assign applications to virtual machines in a data centre with respect to energy usage, CPU utilisation and application completion time which shows good results. For problems where there are more than one parameter to optimise, multi-objective goal attainment optimization has been suggested. This algorithm tries to satisfy a given set of well defined goals subject to multiple constraints. Jones ${ }^{16}$ used the goal attainment algorithm to show how multiple goals regarding supply and demand could be satisfied. Oddoye ${ }^{17}$ has applied goal attainment optimization in the health care sector and shown how it can optimally cut waiting times for patients whilst effectively assigning doctors, nurses and consultants to keep queues to a minimum. Ghoseiri ${ }^{18}$ uses goal attainment to solve the vehicle routing problem and was successful in optimising multiple goals in a reasonable amount of computational time. Multi-objective goal attainment optimization will be used in this paper to solve this "command-to-drive" assignment problem.

Hybrid genetic algorithms ${ }^{19}$ and evolutionary algorithms ${ }^{20}$ have also been used to optimally schedule computing resources over a network where energy was one of the optimisation objectives. However, a weakness is that it did not verify the algorithm on real data.

\section{Multi-Objective Goal Attainment Optimization}

The main problem is to allocate $n$ commands onto $m$ drives for $n \gg m$ in order to achieve the goals of minimal energy usage, fast execution time and 
low storage cost. Let $\mathbf{x}=\left\{x_{i j}\right\}$ be the primary decision variable defined by:

$$
x_{i j}=\left\{\begin{array}{l}
1 \text { if command } i \text { is allocated to drive } j \\
0 \text { otherwise }, \quad i=1, \ldots, n ; \quad j=1, \ldots, m .
\end{array}\right.
$$

138 Energy usage is modelled by considering the energy needed to execute 139 command $i$ on drive $j$. Let $\mathbf{e}=\left\{e_{i j}\right\}$ denote the energy decision variable:

$$
\begin{aligned}
& e_{i j}=\text { energy usage if command } i \text { is assigned to drive } j \\
& \qquad \text { for } i=1, \ldots, n ; \quad j=1, \ldots, m
\end{aligned}
$$

The time to execute command $i$ on drive $j$ is modelled using the decision variable $\mathbf{t}=\left\{t_{i j}\right\}$ where:

$$
t_{i j}=\text { execution time if command } i \text { is assigned to drive } j
$$

$$
\text { for } \quad i=1, \ldots, n ; \quad j=1, \ldots, m \text {. }
$$

142 The storage cost is defined as the percentage of free disk space needed to execute command $i$ on drive $j$. This cost will therefore be smaller on large capacity bulk hard drives but larger on small capacity drives. It is modelled by the decision variable $\mathbf{s}=\left\{s_{i j}\right\}$ where:

$$
\begin{aligned}
& s_{i j}=\text { storage cost if command } i \text { is assigned to drive } j \\
& \qquad \text { for } i=1, \ldots, n ; \quad j=1, \ldots, m .
\end{aligned}
$$

${ }_{46}$ The multi-objective goal attainment model proposed considers energy usage $\mathbf{e}$, execution time $\mathbf{t}$ and storage cost $\mathbf{s}$ and has the form: 


$$
\begin{aligned}
\min G= & w_{e} \sum_{i=1}^{n} \frac{p_{i}^{e}}{E_{i}^{\text {goal }}}+w_{t} \sum_{i=1}^{n} \frac{p_{i}^{t}}{T_{i}^{\text {goal }}}+w_{s} \sum_{j=1}^{m} \frac{p_{j}^{s}}{S_{j}^{\text {goal }}}, \\
\text { subject to } & \sum_{i=1}^{n} e_{i j} x_{i j}+n_{i}^{e}-p_{i}^{e}=E_{\text {goal }}, \text { for } \quad j=1, \ldots, m ; \\
& \sum_{i=1}^{n} t_{i j} x_{i j}+n_{i}^{t}-p_{i}^{t}=T_{\text {goal }}, \text { for } \quad j=1, \ldots, m ; \\
& \sum_{i=1}^{n} s_{i j} x_{i j}+n_{i}^{s}-p_{i}^{s}=S_{\text {goal }}, \text { for } \quad j=1, \ldots, m ; \\
& \sum_{i=1}^{n} t_{i j} x_{i j} \leq T_{\text {max }}, \text { for } \quad j=1, \ldots, n ; \\
& \sum_{i=1}^{n} s_{i j} x_{i j} \leq S_{\text {max }}, \text { for } \quad j=1, \ldots, m ; \\
& \sum_{j=1}^{n} x_{i j}=1, \text { for } \quad i=1, \ldots, n ;
\end{aligned}
$$

The following conditions also hold

$$
\begin{gathered}
x_{i j}=0 \text { or } 1 \quad i=1, \ldots, n ; \quad j=1, \ldots, m \\
n_{i}^{e}, p_{i}^{e} \geq 0 ; n_{i}^{t}, p_{i}^{t} \geq 0 \quad i=1, \ldots, n ; \\
n_{j}^{s}, p_{j}^{s} \geq 0 ; n_{i}^{t}, p_{i}^{t} \geq 0 \quad j=1, \ldots, m ;
\end{gathered}
$$

In this formulation $E_{\text {goal }}, T_{\text {goal }}$ and $S_{\text {goal }}$ denote the goals for energy usage, execution time and storage cost respectively. For each of these goals $n_{i}^{e}, p_{i}^{e}$, $n_{i}^{t}, p_{i}^{t}$ and $n_{j}^{s}, p_{j}^{s}$ denote the positive and negative deviations from the energy, time and storage goals respectively. The constraints $T_{\max }$ and $S_{\max }$ are imposed so that the total time and storage costs respectively for a given set of commands do not exceed these thresholds. This is to ensure that commands are executed within a given time period and that the commands are not all 
issued to solid state drives. The parameters $w_{e}, w_{t}, w_{s}$ are used to weight the importance of each of the goals for energy, time and storage respectively. The final constraint of equation 7 ensures that each command is assigned to exactly one drive.

\section{Experimental Details}

Two different experimental setups were required to gather all the necessary data for this paper. Firstly, a data logger system was designed to capture current and voltage data from individual drives. This setup was necessary to identify the characteristics of each model of drive.

Secondly, another data logger system was created to capture voltage and current data from a much larger drive storage cluster containing multiple drives. This was created in order to test the effectiveness of the proposed method on a real data storage system.

\subsection{Data Logger System for Individual Drives}

The data logger system for an individual drive (see figure 1) consists of:

- One unit of the drive to be tested.

- Two Adafruit INA219 voltage and current sensor boards to measure current and voltage from the drive's $+5 \mathrm{~V}$ and $+12 \mathrm{~V}$ supply lines.

- One Arduino Leonardo controller board for controlling the sensors, collecting samples and sending the data to the computer.

- One desktop computer to run the software to supervise the controller, store the samples and generate the required workloads for the drive. 
Figure 1: Data logger system for an individual drive

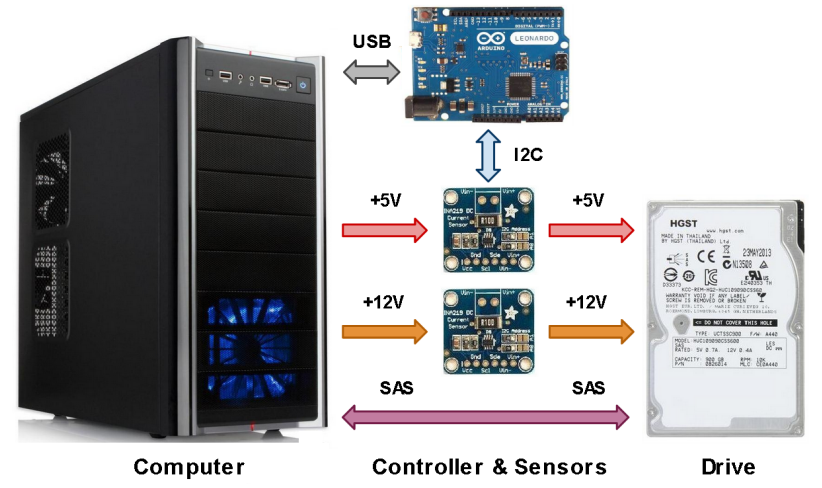

\subsection{Data Logger System for a Drive Cluster}

The data logger for the drive cluster (see figure 2) consists of:

- Thirty-six drives consisting of five different drive types.

- Seventy-two Adafruit INA219 sensor boards (one for each of the two power lines into each of the thirty six drives).

- Twelve Arduino Leonardo controller boards.

- One desktop computer to monitor the controllers, store the sensor data and to provide power for sensors and controllers.

- One high-performance drive cluster populated with the thirty six drives.

- One Hewlett-Packard ProLiant DL360 Generation 5 server to generate workloads.

Additionally the sensors were linked to the controllers via $\mathrm{I} 2 \mathrm{C}$ buses, the controllers were linked to the computer via USB and the storage cluster was linked to the server via a SAS cable. 
Figure 2: Data logger system for a cluster of drives

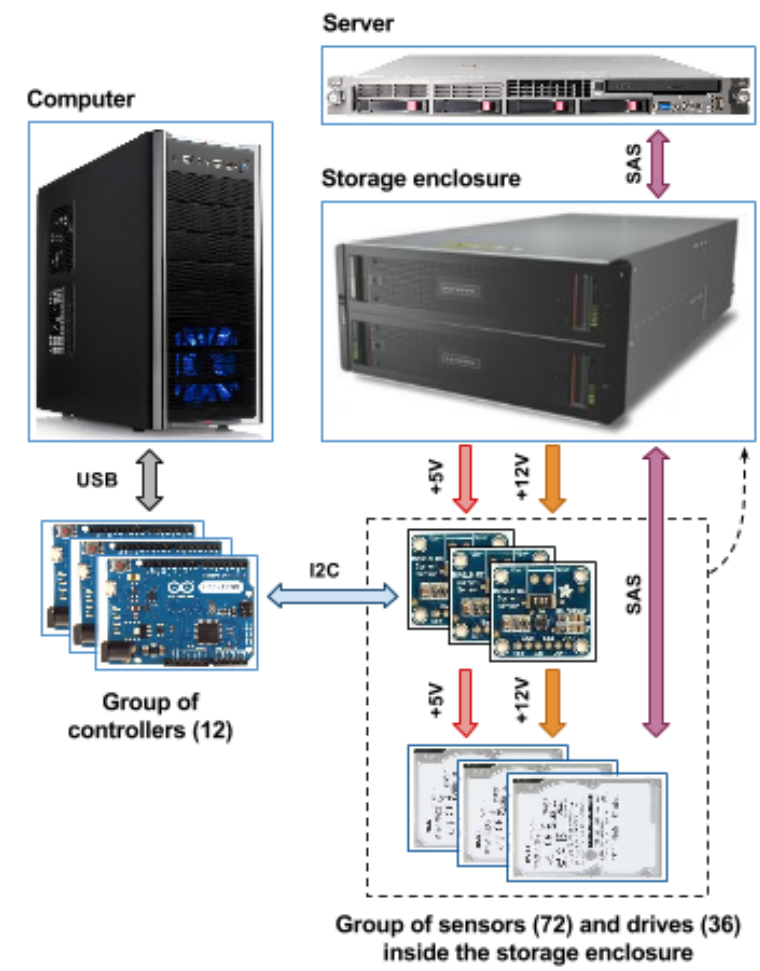

For these experiments, the Arduino IDE programming environment was used to develop the code to control the controller boards. To generate the workloads, the latest version of Iometer was used (version 1.1.0). To analyse the resulting workload and sensor data, Matlab 2014a was used.

Table 1 shows the drives used in the storage cluster, their quantity, their position, their type and their specifications. The drives selected include those types commonly found in a storage cluster. For example, type 5 (high speed SSD), type 1 (fast HDD), types 2 and 3 (medium HDD) and type 4 (slow, bulk storage HDD). The Drive Position column shows where each drive was positioned in the drawer and this number is used to identify drives in the 
Table 1: Details of Drive Types Used

\begin{tabular}{|c|c|c|c|c|}
\hline $\begin{array}{l}\text { Drive } \\
\text { Posi- } \\
\text { tion }\end{array}$ & $\begin{array}{l}\text { Type } \\
\text { Num- } \\
\text { ber }\end{array}$ & $\begin{array}{l}\text { Drive } \\
\text { Type }\end{array}$ & Units & Details \\
\hline $\begin{array}{l}7-12 \\
20\end{array}$ & 1 & HDD & 7 & $\begin{array}{l}\mathrm{SAS}, 73.4 \mathrm{~GB}, 2.5,15 \\
\mathrm{krpm}\end{array}$ \\
\hline $22-24$ & 2 & HDD & 3 & $\begin{array}{l}\mathrm{SAS}, 450 \mathrm{~GB}, 2.5,10 \\
\mathrm{krpm}\end{array}$ \\
\hline $\begin{array}{l}1-6, \\
21\end{array}$ & 3 & HDD & 7 & $\begin{array}{l}\mathrm{SAS}, 900 \mathrm{~GB}, 2.5,10 \\
\mathrm{krpm}\end{array}$ \\
\hline $25-36$ & 4 & HDD & 12 & $\begin{array}{l}\text { SAS, } 3000 \mathrm{~GB}, 2.5,7.2 \\
\mathrm{krpm}\end{array}$ \\
\hline 13-19 & 5 & SSD & 7 & $\begin{array}{llll}\text { SAS, } & 200 \quad \text { GB, } & 2.5, \\
\text { SLC } & & & \end{array}$ \\
\hline
\end{tabular}

results section.

\subsection{Experimental Setup}

In order to prepare the model, it was necessary to understand the characteristics in terms of energy and time of each of the five drive types. Two different command sets (workloads) were created in order to test the model's performance. Throughout this paper, they will be referred to as the 'Standard' command set and the 'File Server' command set.

\subsubsection{Description of Commands}

In this section, the types of command used will be described and explained. The term 'Data Transfer Size' (e.g. 4096B) refers to the amount of data than is being either read from a drive or written onto a drive. 
The term 'Read Access' refers to the percentage of data that is being read from the drive. A value of $100 \%$ means all of the data is being read. A value of $50 \%$ means that half of the data is being read from the drive and the other half is being written. A value of $0 \%$ means that no data is being read so all the data is being written onto the drive.

Files can be stored on a drive either sequentially or in multiple sectors in different areas of the drive. Unsurprisingly, it is preferable to store data in contiguous locations than random locations as it uses less energy and takes less time to complete. This is because if a file is stored in random locations, the head (in a HDD) must move more times to access all parts of the file. The term 'Random Access' refers to the percentage of data that is read/written at random. A value of $100 \%$ means that all data is being read/written from random locations. A value of $50 \%$ means that half of the data is being $\mathrm{read} /$ written from random locations and the other half is being read/written from sequential locations. A value of $0 \%$ means all data is being read/written from sequential locations.

\subsection{2. 'Standard' Command Set}

Twenty-four commands were selected to form the 'Standard' command set and details of these commands can be found in table 2. Each of these commands was repeatedly executed on each drive model for 60 seconds over 3 separate tests. The average energy and time taken to execute each command was computed and details can be found in figures 3 and 4 respectively. 
Table 2: Specifications of standard commands to be executed

\begin{tabular}{|c|c|c|c|}
\hline $\begin{array}{l}\text { Profile } \\
\text { Num- } \\
\text { ber }\end{array}$ & $\begin{array}{l}\text { Data Trans- } \\
\text { fer Size [B] }\end{array}$ & $\begin{array}{l}\text { Read Ac- } \\
\text { cess }[\%]\end{array}$ & $\begin{array}{l}\text { Random Ac- } \\
\text { cess }[\%]\end{array}$ \\
\hline 1 & 512 & 100 & 100 \\
\hline 2 & 512 & 50 & 100 \\
\hline 3 & 512 & 0 & 100 \\
\hline 4 & 512 & 100 & 0 \\
\hline 5 & 512 & 50 & 0 \\
\hline 6 & 512 & 0 & 0 \\
\hline 7 & 4096 & 100 & 100 \\
\hline 8 & 4096 & 50 & 100 \\
\hline 9 & 4096 & 0 & 100 \\
\hline 10 & 4096 & 100 & 0 \\
\hline 11 & 4096 & 50 & 0 \\
\hline 12 & 4096 & 0 & 0 \\
\hline 13 & 65536 & 100 & 100 \\
\hline 14 & 65536 & 50 & 100 \\
\hline 15 & 65536 & 0 & 100 \\
\hline 16 & 65536 & 100 & 0 \\
\hline 17 & 65536 & 50 & 0 \\
\hline 18 & 65536 & 0 & 0 \\
\hline 19 & 1048576 & 100 & 100 \\
\hline 20 & 1048576 & 50 & 100 \\
\hline 21 & 1048576 & 0 & 100 \\
\hline 22 & 1048576 & 100 & 0 \\
\hline 23 & 1048576 & 50 & 0 \\
\hline 24 & 1048576 & 0 & 0 \\
\hline
\end{tabular}


Figure 3: Typical Command Energy Consumption of Each of the Drive Types for the Standard Command Set

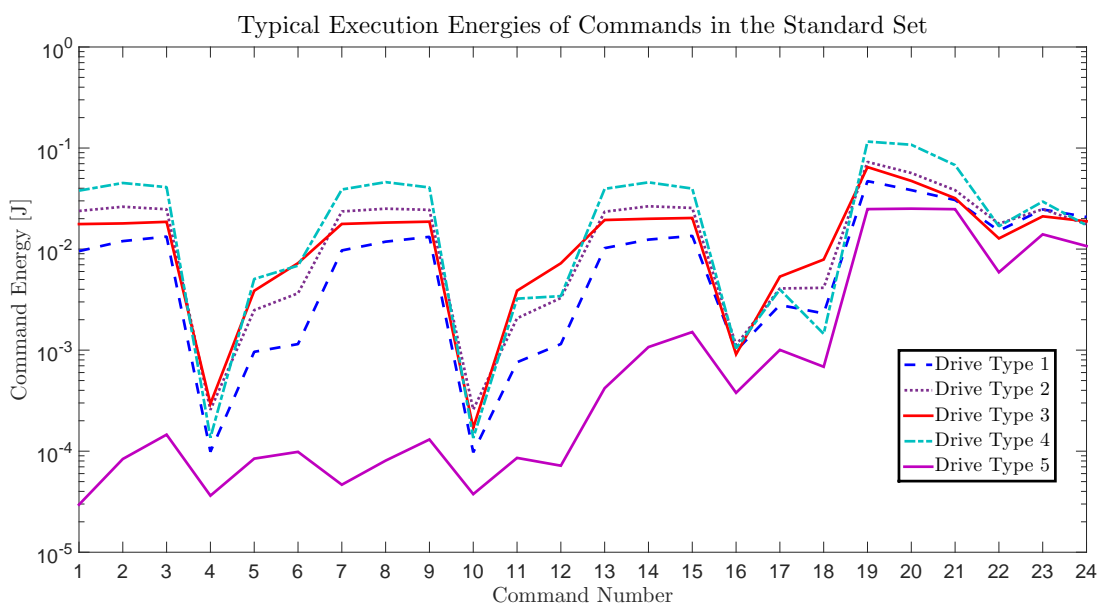

Figure 4: Typical Command Time Execution of Each of the Drive Types for the Standard Command Set

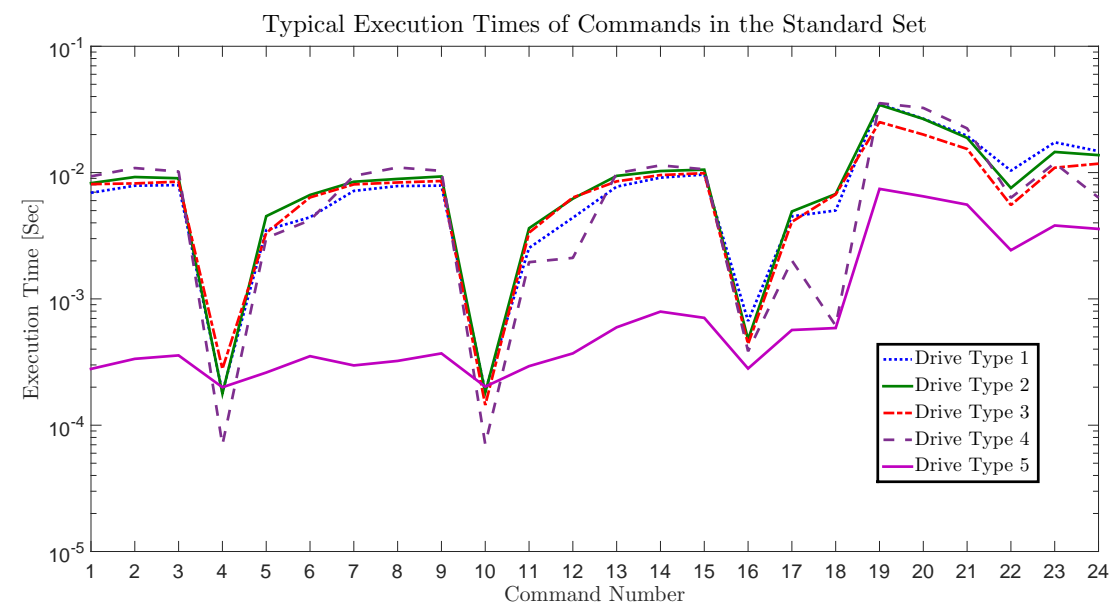

\subsubsection{File Server Command Set}

A set of commands typically found in file server applications (see table 3) was also constructed. This was based on a typical file server access pattern as defined by Intel $^{21}$. Three workloads were executed comprising of 100 
commands in the proportions given in table 3 on each of the drive types for 60 seconds. The average energy used and time taken to execute one command were computed and can be seen in figures 5 and 6 .

This data was used to populate an energy usage and execution time lookup table that forms the basis for the model. Figure 3 shows the average energy usage for all twenty-four commands executed on the five different drive types. The average execution times are shown in figure 4 . In order to present the data, the scale on the $\mathrm{y}$-axis was made logarithmic so it was possible to compare the solid state type 5 drive with the other 4 hard disks. Figures 5 and 6 show the execution energies and times for the File Server commands respectively. The energy and time graphs clearly show that the solid state drive offers superior energy consumption and execution times. To ensure a balanced comparison, a 'storage cost' is introduced (for the definition, see section 3). This variable will be small for the large capacity drives such as the type 4 but larger for the type 1 and type 5 drives. A weighting towards this variable will ensure that not all commands are repeatedly assigned to the solid state drive. This will make the model more realistic.

\subsection{Idle Data Collection}

The vast majority of energy usage during drive operation occurs in simply providing power to the electronics ( +5 volt line on SSD and HDD) and in keeping the platters spinning and the arm moving (+12 volt line HDD only). The drives themselves have their own in-built intelligence, which acts to check the integrity of data written. On traditional hard disks, a Background Media Scan (BMS) can take place to check for errors by reading previously written data. This activity usually takes place during idle periods, depending on 
Figure 5: Typical Command Energy Consumption of Each of the Drive Types for the File Server Commands

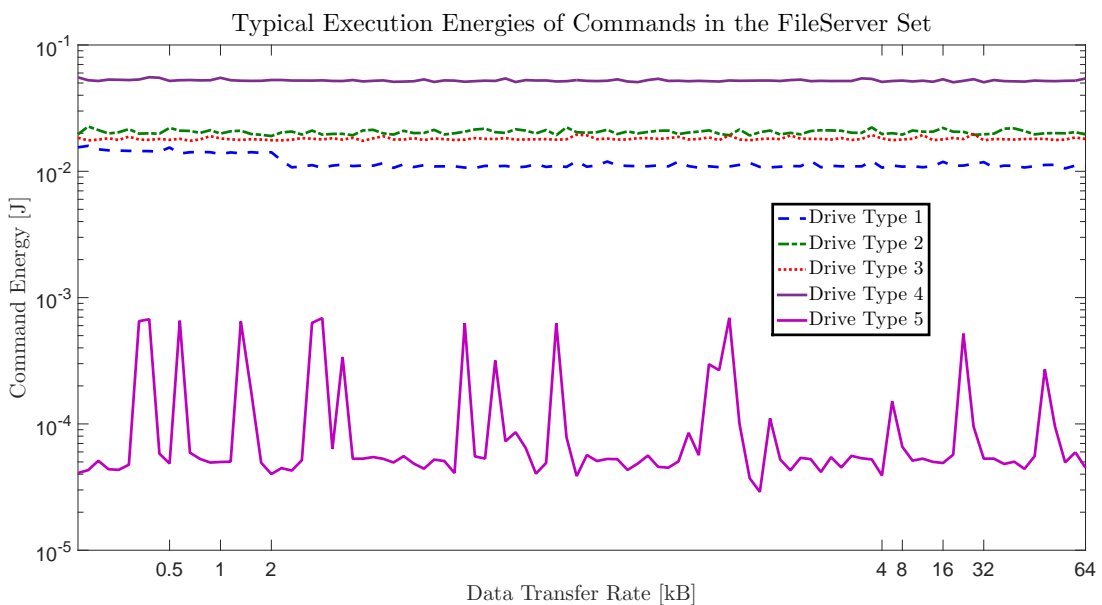

Figure 6: Typical Command Time Execution of Each of the Drive Types for the File Server Commands

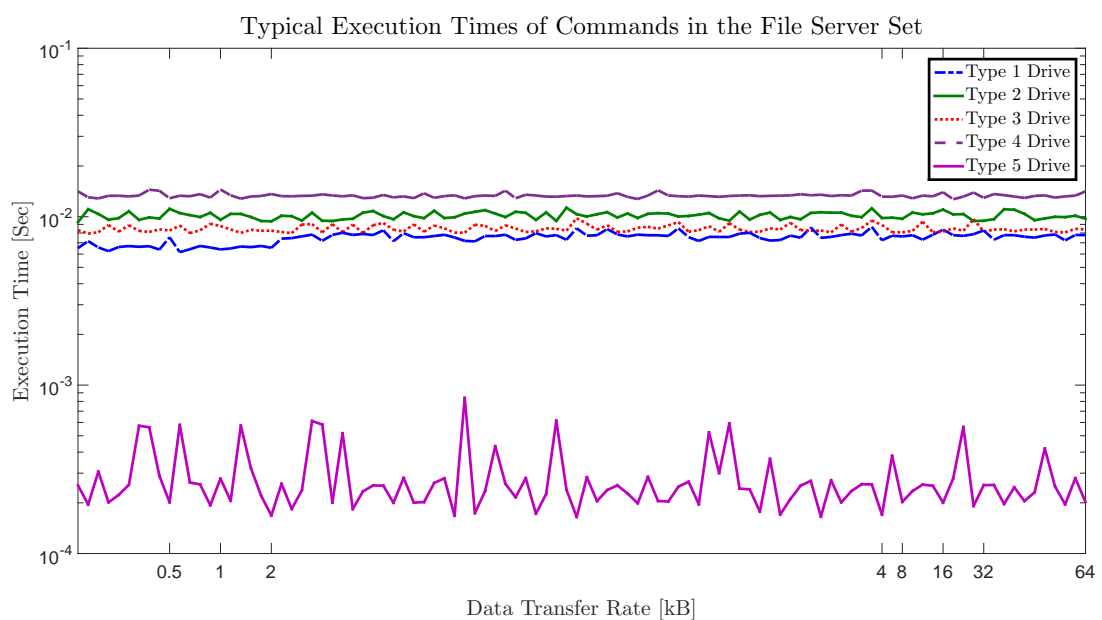

how much data has been written. On certain hard disk types, data is checked immediately after writing. This activity is known as Idle Read After Write (IRAW). Solid state drives perform an activity known as Wear-levelling Maintenance (WLR) to ensure erase/write cycles are evenly distributed across all 
Table 3: Specifications of File Server commands to be executed

\begin{tabular}{|c|c|c|c|}
\hline $\begin{array}{l}\text { Percentage } \\
\text { of Total } \\
{[\%]}\end{array}$ & $\begin{array}{l}\text { Data } \\
\text { Transfer } \\
\text { Size }[k B]\end{array}$ & $\begin{array}{l}\text { Read } \\
\text { Access }[\%]\end{array}$ & $\begin{array}{l}\text { Random } \\
\text { Access [\%] }\end{array}$ \\
\hline 10 & 0.5 & 80 & 100 \\
\hline 5 & 1 & 80 & 100 \\
\hline 5 & 2 & 80 & 100 \\
\hline 60 & 4 & 80 & 100 \\
\hline 2 & 8 & 80 & 100 \\
\hline 4 & 16 & 80 & 100 \\
\hline 4 & 32 & 80 & 100 \\
\hline 10 & 64 & 80 & 100 \\
\hline
\end{tabular}

locations. These maintenance activities caused difficulty when attempting to accurately measure the energy required for a given set of commands or for a given power mode (active or idle). As such, data collected regarding idle state and command energies was collected after these maintenance activities were completed. Not all power states could be observed (or even detected) during a 24 hour idle period that the drives were subjected to but the observed power states are listed. For the purposes of the model, it was assumed that if a drive is not executing commands, it is in the IDLE A power state (HDD) or the IDLE state (SSD). Table 4 provides details of the average power on the $+5 \mathrm{~V}$ and $+12 \mathrm{~V}$ supply lines for each observed drive state. Not all states (according to manufacturer guides) were observed and it was not possible to 'force' a drive into all specific states during this research. The single drive data logger was used to gather this information.

For the HDD's, the IDLE A state occurs when the disks are spinning 
at full speed. Some electronics are turned off but the heads are loaded so commands can be received and processed immediately. The IDLE B state occurs when the disks are spinning at full speed but the heads are unloaded. In the IDLE C state, the disks are spinning at a reduced speed and the heads are again unloaded. In the STANDBY state, the disks do not spin and the heads are unloaded but commands can still be received (though the drive will take a while to get up to speed). The SLEEP state is a slightly lower power level than STANDBY.

The idle data analysis shows that significant energy savings can be made over time if it is possible to put drives into lower power states without compromising the performance of the storage cluster. For example, if a Type 4 disk spends 2 hours in IDLE B instead of IDLE A state, the energy saved is approximately 11,124 Joules (20\%).

\section{Proposed Method}

Through preliminary experiments, it was found that a time interval of 10 milliseconds was a suitable interval between consecutive optimisations for the File Server commands as well as the Standard commands for the drive cluster system. If the time interval was too low, then there would be too many commands to be assigned and not enough drives to make the assignment.

In the proposed model, the drives were modelled with a command queue and without. For this model, if a drive has a command queue, it is able to continue to be assigned commands until the queue is full. If it doesn't have a command queue, it must wait until it has executed its current command before it can be assigned another command. 
Table 4: Details of Detected Drive States

\begin{tabular}{|c|c|c|c|c|c|}
\hline Type & Drive & Power $\quad(+5 \mathrm{~V}$ & Power $\quad(+12 \mathrm{~V}$ & Total & Idle State \\
\hline Number & Type & line) $[\mathrm{W}]$ & line) $[\mathrm{W}]$ & Power & \\
\hline 1 & HDD & 5.004 & 2.183 & 7.187 & Active \\
\hline 1 & HDD & 3.437 & 2.201 & 5.638 & IDLE A \\
\hline 2 & HDD & 3.640 & 2.943 & 6.583 & Active \\
\hline 2 & HDD & 1.739 & 2.948 & 4.687 & IDLE A \\
\hline 3 & HDD & 3.010 & 2.080 & 5.089 & BMS \\
\hline 3 & HDD & 1.983 & 2.302 & 4.284 & IDLE A \\
\hline 3 & HDD & 1.361 & 1.678 & 3.038 & IDLE B \\
\hline 4 & HDD & 2.795 & 5.762 & 8.557 & BMS \\
\hline 4 & HDD & 1.743 & 5.868 & 7.611 & IDLE A \\
\hline 4 & HDD & 1.349 & 4.717 & 6.066 & IDLE B \\
\hline 5 & SSD & 3.212 & 1.725 & 4.937 & IDLE \\
\hline
\end{tabular}

As the proposed method is new (to the best of the author's knowledge), there are no other algorithms or methods that operate so deep within storage clusters at the file system level in this manner. The other methods listed in section 2 focus on a higher level, whereby a drive can be put into a different energy saving state depending on its level of activity. For this reason, the multi-objective goal attainment algorithm was compared against a purely random assignment of commands to drives at each round of optimisation. If drive $d_{i}$ is executing command $c_{j}$, it cannot execute another until time $t_{i j}$ has passed, where $t_{i j}$ is the time taken to execute command $c_{j}$ on disk $d_{i}$. For example, if a command takes 31 milliseconds to execute on a given drive and this command is assigned during optimisation round $p$, then this drive is unavailable until optimisation round $p+4$. The experiment was carried 
out as follows:

- With all 36 drives available for assignment, perform the first round of optimisation.

- Assign commands to drives using the optimisation results.

- Identify all $36-n$ free drives where $n$ is the number of busy drives and perform the next round of optimisation.

- Continue until all rounds of optimisation are complete.

\subsection{Goal Attainment Algorithm Parameters}

The setting up of these experiments required a fine balance between achieving a feasible solution and the assignment that offered the lowest energy consumption. In section 3 , there are parameters $T_{\max }$ and $S_{\max }$ to set. Parameter $T_{\max }$ is a hard limit on the total amount of time it takes for any HDD/SDD to execute commands assigned to it. For these experiments, $T_{\max }$ was set at 36 milli-seconds. If the value was set too small, the optimisation problem was infeasible whereas if it was set too large, the algorithm would find sub-optimal solutions. Parameter $S_{\max }$ is a limit on the maximum storage cost per drive and is set at 1.000001. The decimal component refers to the maximum proportion of free drive space that data from an executed (write) command may occupy (i.e. 0.0001\%). This ensures that for example large write commands are issued to drives with the biggest amounts of free space.

The weights are set in the proportion of 100:100:1 for energy, time and storage respectively as energy and time are deemed more important than storage. The goals are set as 0.0005:0.0005:0 for energy (joules), time (seconds) 
and storage (proportion of free space) respectively. Preliminary experimentation showed it was best to set goals that were slightly unobtainable rather than dynamically using the minimum possible values for a given command set.

\subsection{Standard Commands Experiment}

The model was run to simulate 60 seconds of drive cluster activity, which meant that $60 / 0.01=6,000$ rounds of optimisation would be carried out for the Standard commands. A set of random commands was collected from the Standard command set (see table 2). For each of the 6,000 rounds of optimisation, 10 commands were chosen at random from the selection of 24 . Duplicates were allowed. This meant in total that 60,000 random commands were optimised. Five different random command datasets were created in order to validate the performance of the model. This was to ensure that the performance of the algorithm was consistent and un-biased. The average energy consumption from these command datasets was reported and was found to be remarkably consistent with a standard deviation of less than $0.03 \%$.

\subsection{File Server Commands Experiment}

The model was again run to simulate 60 seconds of file server activity as defined by Intel $^{21}$, which meant that $60 / 0.01=6,000$ rounds of optimisation would be carried out for the File Server commands. Table 3 shows that there were 8 unique commands. In order to dynamically model a typical file server, a set of 100 commands was created, which consisted of the 8 unique commands in the proportions given in the first column of this table. For each 
of the 6,000 rounds of optimisation, 10 commands were chosen at random from this selection of 100. Duplicates were allowed. This meant in total that 60,000 random commands were optimised. Five different random command datasets were created in order to validate the performance of the model. The average energy consumption from these command datasets was reported and was found to be remarkably consistent with a standard deviation of less than $0.03 \%$. Again, this was to ensure that the performance of the algorithm was consistent and un-biased.

\section{Results}

In this section, the energy results for both the Standard commands and the File Server commands are presented and analysed in depth. A brief summary is presented in table 5. The method shows clear energy savings for both workloads, particularly for the File Server, regardless of whether the drives have a queue or not, over a purely random assignment. Note that 'command' energy refers to the energy used by the drive to specifically execute the commands. Total energy is defined as command energy plus idle energy.

\subsection{File Server Command Set Results}

\subsubsection{Without Queue}

Using all 36 drives in the IDLE A state for 60 seconds required a total idle energy consumption of 12,565 Joules. By assigning commands randomly, the total energy consumption was 15,713 Joules (3,148 Joules of command energy). However, by optimally assigning commands using the goal attainment 
Table 5: Overall Results Summary

\begin{tabular}{lllll}
\hline $\begin{array}{l}\text { Workload } \\
\text { Set }\end{array}$ & $\begin{array}{l}\text { Queue } \\
\text { mave. } \\
\text { mand } \\
\text { Saving [\%] }\end{array}$ & $\begin{array}{l}\text { Com- } \\
\text { Energy }\end{array}$ & $\begin{array}{l}\text { Ave. } \\
\text { Energy } \\
{[\%]}\end{array}$ & $\begin{array}{l}\text { Overall } \\
\text { Saving }\end{array}$ \\
\hline File Server & Yes & 84.88 & 16.37 \\
\hline File Server & No & 86.25 & 17.41 \\
\hline $\begin{array}{l}\text { Standard } \\
\text { Com- }\end{array}$ & & 15.97 & 1.05 \\
mands & & & \\
\hline $\begin{array}{l}\text { Standard } \\
\text { Com- }\end{array}$ & & 13.27 & 1.31 \\
mands & & & \\
\hline
\end{tabular}

method, the total energy consumption was only 12,978 Joules (433 Joules of command energy). This resulted in a huge energy saving of around $86 \%$ for command energy and $17 \%$ in total (see Figure 7 ). The smooth gradient of the optimised line in figure 7 shows that the energy savings were made continuously throughout each round of optimisation, whatever random set of commands were chosen.

Figure 8 compares the number of commands that were assigned to each of the 36 drives using the optimised and the random assignment and unsurprisingly, the majority of commands have been assigned to drive types 5 (low energy solid state), type 1 (fastest HDD) and type 3 (next lowest energy HDD).

Figure 9 shows a comparison between the command energy consumption for each drive type using the optimised and random assignments. As expected, there are large energy savings (nearly 1,000 Joules) to be made by 
Figure 7: Command Energy Consumption of the Drives using an Optimised or Random Assignment

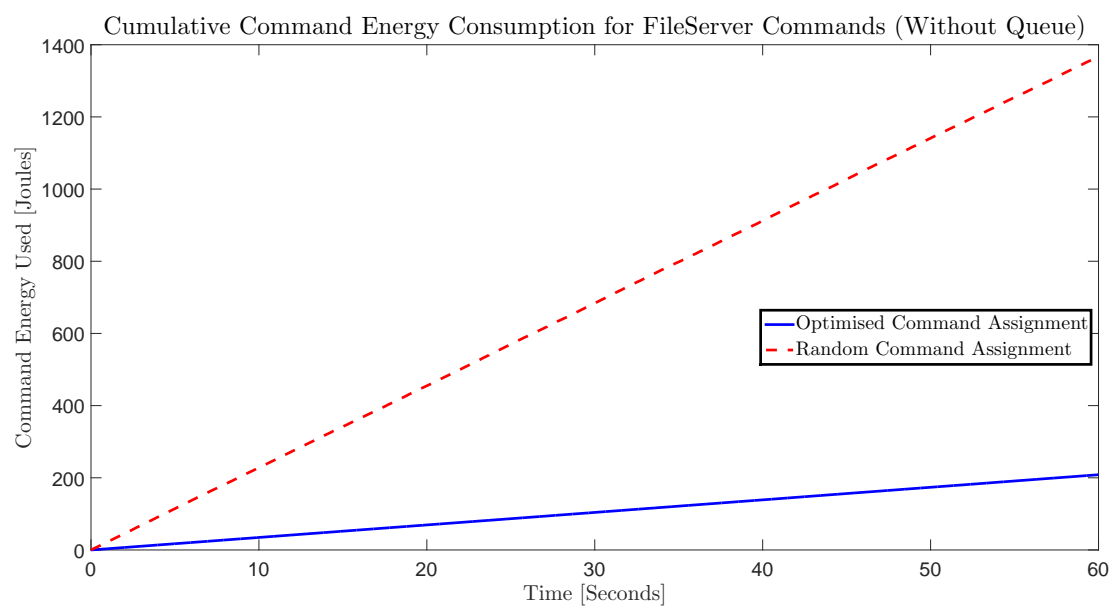

not assigning commands to drive types 2 and 4 . Interestingly it shows that drive type 5 is using more energy in the optimised assignment but this is because it has received many more commands to execute.

Figure 10 shows an illustrated example of the distribution of the different types of commands assigned to drive types 1, 3 and 5 using the optimised method. It shows that commands with very small data transfer sizes $(\leq 4$ $\mathrm{kB}$ ) are mostly executed by type 5 drives whereas for File Server commands with larger data transfer rates $(\geq 4 \mathrm{kB})$, type 1 drives are preferred. In this experiment, the type 5 drives received the maximum possible allocation of commands (1 command per drive per iteration) so it shows that if the type 5 drives are not available then the type 1 disks are the next best, followed by type 3 .

Table 6 shows the percentages of commands that have been assigned to each drive type unit. The random assignment column will not have roughly 
Figure 8: Number of Commands Assigned to Each of the Drives using an Optimised or Random Assignment

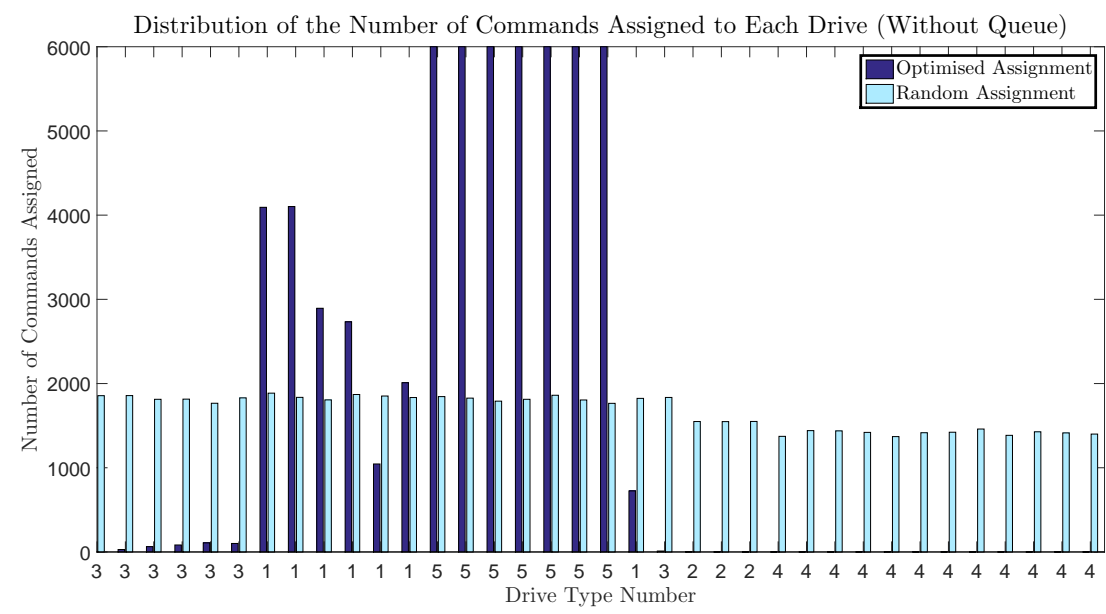

Figure 9: Command Energy Consumption for Each Drive Type using an Optimised or Random Assignment

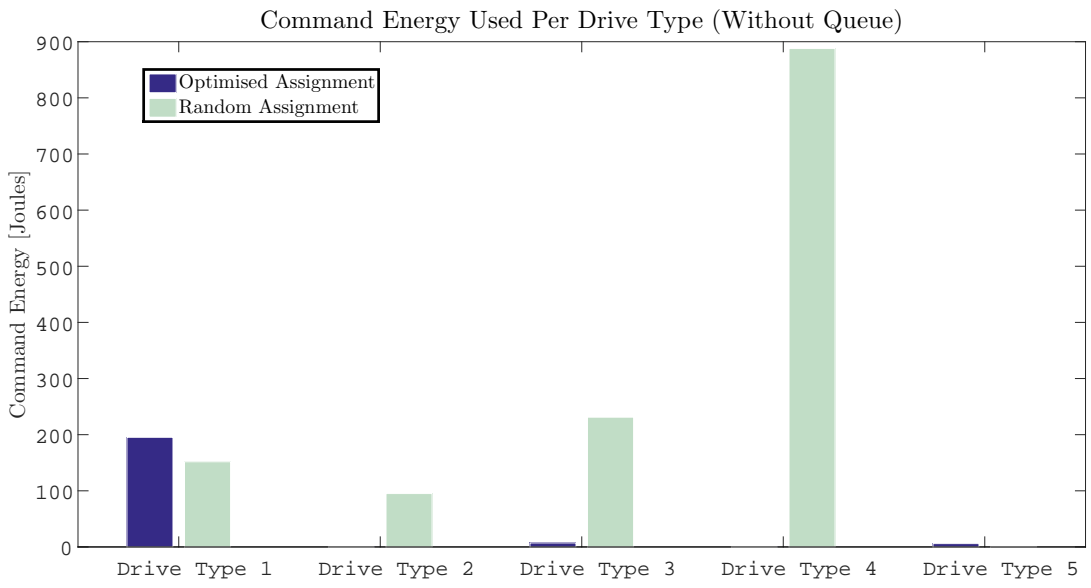

equal proportions of commands assigned to each unit as some drives can execute commands faster than others so they will be available for assignment more often. It shows clearly how drive type 5 was favoured for its low energy properties. 
Figure 10: Distribution of Optimised Command Assignments to Drive Types 1, 3 and 5

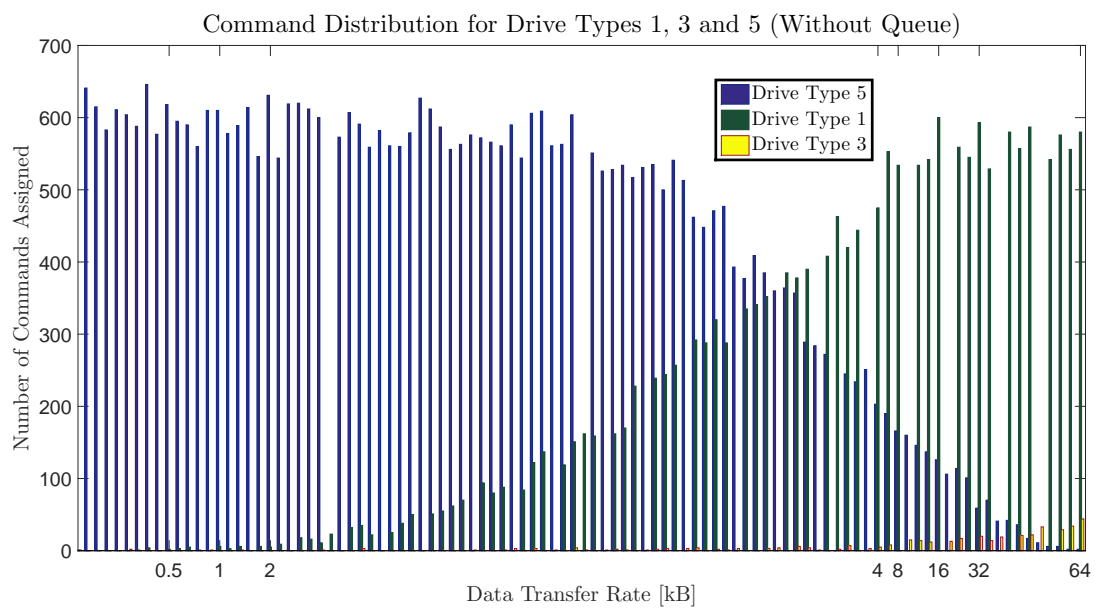

\subsubsection{With Queue}

Using all 36 drives in the IDLE A state for 60 seconds required a total energy consumption of 12,565 Joules. By assigning commands randomly, the total energy consumption was 15,567 Joules (3,002 Joules of command energy). However, by optimally assigning commands using the goal attainment method, the total energy consumption was only 13,019 Joules (454 Joules of command energy). This resulted in a huge energy saving of around $85 \%$ for command energy and $16 \%$ in total. The figures for the results in this section will not be included here as they are almost identical to those in the 'Without Queue' subsection.

The key differences between the optimisations without a queue and with a queue can be seen in table 7 . When the drives are modelled with a queue, the random assignment doesn't favour the type 5 drives as much because the other drive types are available for longer periods (as the queue allows more commands to be assigned even if the current ones haven't yet been 
Table 6: Proportion of Commands Assigned per Drive Type Unit

\begin{tabular}{llll}
\hline $\begin{array}{l}\text { Drive } \\
\text { Type } \\
\text { Number }\end{array}$ & $\begin{array}{l}\text { Optimised } \\
\text { Assign- } \\
\text { ment }[\%]\end{array}$ & $\begin{array}{l}\text { Random } \\
\text { signment }[\%]\end{array}$ & $\begin{array}{l}\text { As- } \\
\text { Difference } \\
{[\%]}\end{array}$ \\
\hline 1 & 4.27 & 2.80 & 152.50 \\
\hline 2 & 0.00 & 2.40 & -100.00 \\
\hline 3 & 0.01 & 2.80 & -99.64 \\
\hline 4 & 0.00 & 2.18 & -100.00 \\
\hline 5 & 10.00 & 3.92 & 255.10 \\
\hline
\end{tabular}

Table 7: Proportion of Commands Assigned per Drive Type Unit for the File Server Commands With Queue

\begin{tabular}{llll}
\hline $\begin{array}{l}\text { Drive } \\
\text { Type } \\
\text { Number }\end{array}$ & $\begin{array}{l}\text { Optimised } \\
\text { Assign- } \\
\text { ment }[\%]\end{array}$ & $\begin{array}{l}\text { Random } \\
\text { signment }[\%]\end{array}$ & $\begin{array}{l}\text { Percentage } \\
\text { Difference } \\
{[\%]}\end{array}$ \\
\hline 1 & 4.19 & 3.07 & 36.48 \\
\hline 2 & 0.00 & 2.58 & -100.00 \\
\hline 3 & 0.09 & 3.04 & -97.04 \\
\hline 4 & 0.00 & 2.36 & -100.00 \\
\hline 5 & 10.00 & 3.02 & 331.13 \\
\hline
\end{tabular}

executed). This is why the random assignment uses more energy because the higher energy disks are more available. However, there is little difference between the optimisation results with or without a queue.

\section{2. 'Standard' Command Set Results}

\subsubsection{Without Queue}

Optimising the Standard command set posed a different set of challenges as there was a larger variation in the properties of the different commands 
Figure 11: Command Energy Consumption of the Drives using an Optimised or Random Assignment

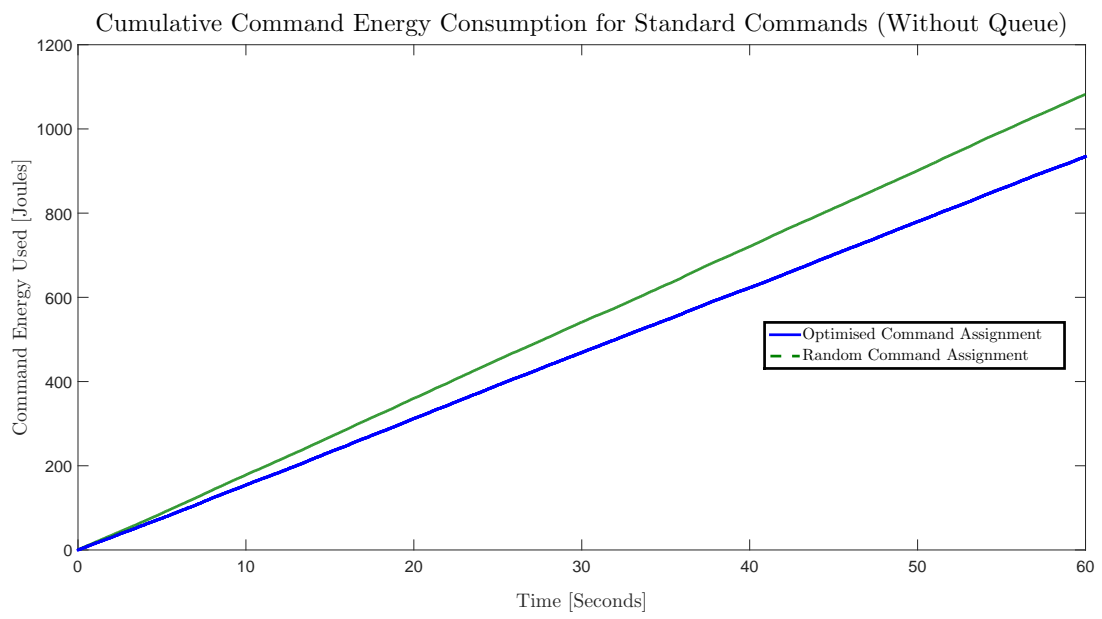

compared to the File Server set. For example, writing a 1MB file on an SSD or a HDD will be slower and will use more energy than executing a 512B read command.

The idle energy used by this model was 12,565 Joules. Using the optimised method, the average total energy consumption was 13,506 Joules (941 Joules of command energy) across the five random command sets with a standard deviation of 4 Joules. Using the random assignment method, the average total energy consumption was 13,650 Joules (1,085 Joules of command energy) across the five random command sets with a standard deviation of 3 Joules. This was a saving of around $13 \%$ in terms of command energy and around $1 \%$ in total. Again, figure 11 shows that the energy savings through optimisation were made throughout.

Figure 12 shows how many commands were assigned to each of the 36 drives using the random and optimised methods. Type 5 drives (SSD) have 
Figure 12: Assignment of Commands to Drives using an Optimised or Random Assignment

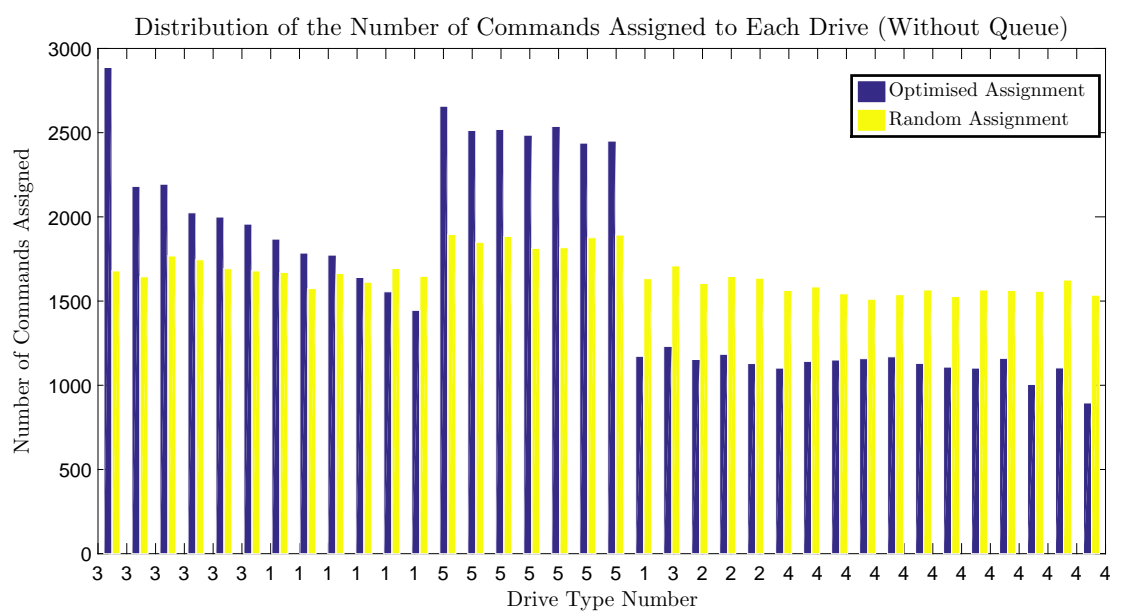

more random commands assigned to them by virtue of the fact that on average they can execute commands faster so they are available for assignment more often. It shows that the optimised method makes energy savings by assigning more commands to drive types 5, 3 and 1 whilst assigning less to drive types 2 and 4 . This is logical given that the SSDs (Drive Type 5) offer much lower execution times and lower energy costs. Drive types 2 and 3 are similar in terms of platter speed and capacity but as figures 3 and 4 show, drive type 3 has a lower energy consumption and execution time for the majority of these commands so the model assigned more commands to type 3 drives. Drive type 4 is the slow speed, large capacity hard disk and typically has the highest energy consumption. However, it does have surprisingly fast execution times (see figure 4), which means that commands are still assigned to it.

Figure 13 shows the distribution of commands from the Standard command set to drives without a queue using the optimised and random methods. 
Figure 13: Distribution of Commands to Drives using an Optimised or Random Assignment

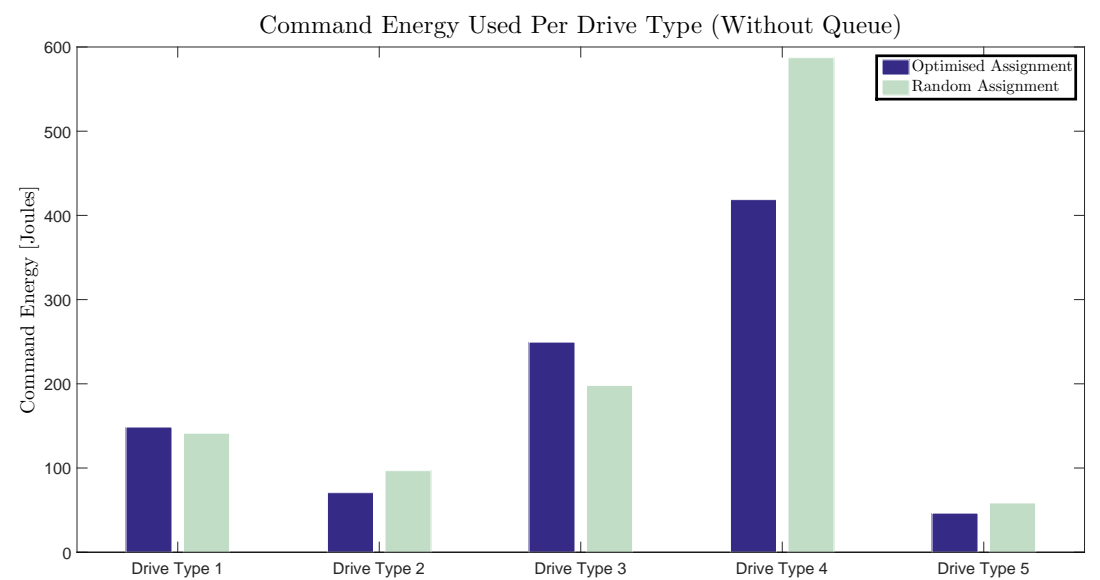

The main energy savings are made by assigning fewer commands to drive types 2 and 4 . Drive type 5 uses more energy in the optimised assignment because it is receiving many more commands.

Figure 14 shows exactly which commands were assigned to drive types 4 and 5 . Although the type 5 drives (SSD) offer superior energy performance over nearly all commands, the superiority is biggest for the $512 \mathrm{~B}$ commands (command numbers 1-6) so they are mainly assigned to this drive type. The frequency and type of commands is too great to just assign everything to type 5 drives and so the type 4 drives are also used, mainly on commands with higher data transfer rates (64 kB and $1 \mathrm{MB}$, commands 13-24). This provides clear insight into exactly how the model made its assignments.

Table 8 shows the percentages of commands assigned to each drive type unit. The random assignment has not assigned an equal proportion of commands to each drive type unit because there is no queue and so as some 
Figure 14: Distribution of Optimised Command Assignments to Drive Types 4 and 5

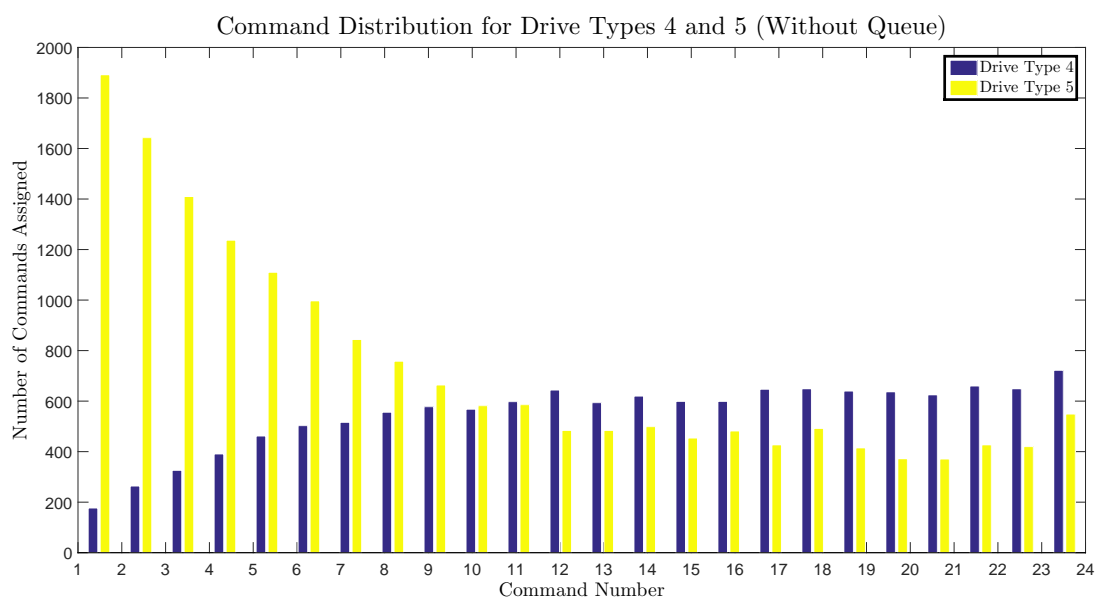

drives execute commands faster than others, the random assignment will be uneven. As expected, drive types 3 and 5 receive more commands at the expense of drive types 2 and 4 .

\subsubsection{With Queue}

The presence of a drive command queue makes no difference to the amount of idle energy consumed over a fixed period so for this model, the amount of idle energy used was 12,565 Joules. Using the optimised method, the average total energy consumption was 13,507 Joules (942 Joules of command energy) over the 5 random command datasets with a standard deviation of 1 Joule. Using the random assignment method, the average total energy consumption was 13,686 Joules (1,121 Joules of command energy) across the five random command sets with a standard deviation of 3 Joules. This was a command energy saving of $16 \%$ and a total energy saving of $1 \%$. It is interesting to note that for the Standard command set, the presence of the queue makes barely any difference on the optimised energy consumption. 
Table 8: Proportion of Commands Assigned per Drive Type Unit

\begin{tabular}{llll}
\hline $\begin{array}{l}\text { Drive } \\
\text { Type } \\
\text { Number }\end{array}$ & $\begin{array}{l}\text { Optimised } \\
\text { Assign- } \\
\text { ment }[\%]\end{array}$ & $\begin{array}{l}\text { Random } \\
\text { signment }[\%]\end{array}$ & $\begin{array}{l}\text { Asercentage } \\
\text { Difference } \\
{[\%]}\end{array}$ \\
\hline 1 & 2.68 & 2.74 & -2.22 \\
\hline 2 & 1.93 & 2.71 & -28.78 \\
\hline 3 & 3.45 & 2.84 & 21.48 \\
\hline 4 & 1.84 & 2.59 & -28.96 \\
\hline 5 & 4.19 & 3.10 & 35.16 \\
\hline
\end{tabular}

Table 9: Proportion of Commands Assigned per Drive Type Unit

\begin{tabular}{llll}
\hline $\begin{array}{l}\text { Drive } \\
\text { Type } \\
\text { Number }\end{array}$ & $\begin{array}{l}\text { Optimised } \\
\text { Assign- } \\
\text { ment [\%] }\end{array}$ & $\begin{array}{l}\text { Random } \\
\text { signment }[\%]\end{array}$ & $\begin{array}{l}\text { Percentage } \\
\text { Difference } \\
{[\%]}\end{array}$ \\
\hline 1 & 2.68 & 2.78 & -3.60 \\
\hline 2 & 1.95 & 2.72 & -28.31 \\
\hline 3 & 3.49 & 2.80 & 24.64 \\
\hline 4 & 1.82 & 2.78 & -34.53 \\
\hline 5 & 4.16 & 2.78 & 49.64 \\
\hline
\end{tabular}

However, by having a queue, it means the drives are more available to receive commands than without so there is more opportunity for random commands to be assigned to high energy drives (e.g. type 4), resulting in higher energy use.

As with the File Server results, the charts for the model with a queue are very similar to the charts for the model without a queue. The main differences are shown in table 9 . The optimised percentage per unit assignments are almost identical with or without a queue (tables 8 and 9) but in the presence 
of a drive queue, where drives are available for assignment more often, the random assignment is roughly equal the same across all drive types, which is why the random assignment uses more energy when the model is run with a queue.

\subsection{Algorithm Performance Impact Implications}

The research presented in this paper illustrates a proof of concept; namely that it is possible to make energy savings deep within the kernel via the optimal allocation of commands. A future working algorithm that is based on the discoveries made in this research will have an energy and time cost and as such, it is not proposed to run such an algorithm real time. This is because it would need to make decisions within nano-seconds in order to ensure overall file system performance is not impaired. Were it to be implemented, it would likely operate offline at fixed periods. Possible fast meta-heuristic algorithms include tabu search $\left({ }^{22}\right)$ and ant colony optimisation $\left({ }^{23}\right)$.

The key discovery is that the research shows where energy savings can be made and further work is needed to convert these insights into a practical application.

\section{Conclusion}

The results show clearly that the model is effective in achieving a reduction in energy consumption regardless of whether the drives have command queue or not. The energy savings are far larger for the File Server command set but this is because commands from this set can be executed far quicker and so the less energy efficient drives (types 2 and 4) are barely used as the other drives have enough capacity to process them. 
Although the Standard command set is not particularly representative of a real world workload, it was selected as it contains a wide range of different command types that can illustrate more clearly how the model performs. Whilst it is well known that type 5 drives (SSD) use less energy than traditional mechanical hard disks, the paper shows that the gap is biggest for the 512B commands so the model mainly assigned these commands to the type 5 drives.

More significantly, it shows how further energy savings can be made by putting drives into lower power states. By showing which types of command should be assigned to a given drive type, it shows that depending on the current workload, some drives can be safely put into lower power states. To illustrate the energy saving potential of this action, consider the File Server 'with queue' experiment. The total command energy saving over the random assignment was 2,548 Joules. If all twelve of the type 4 drives were put into IDLE B mode (instead of IDLE A) for 60 seconds (see table 4), an extra 1,112 Joules would be saved.

Currently, the proposed approach shows a proof of concept and insights into how an intelligent file allocation system might work. It is true that in a future system, the overheads due to this proposed optimisation would need to be balanced against energy savings. The key contribution of this paper is the insight into which commands are best suited to a given drive, rather than how to create a working algorithm. Future research will investigate what impact the proposed method has in terms of overheads but also whether it is best to have a real time system, or whether it is best to periodically perform this optimisation. 


\section{Acknowledgment}

The authors would like to thank and acknowledge financial support from Innovate UK (formerly known as the Technology Strategy Board) under grant number 131195.

\section{Appendix A. Data Sets Used}

The data for the experiments detailed in this paper is presented in the appendix. Time and energy data is presented for both the Standard command set and the File Server command set. Storage costs are not directly presented as they depend on the used capacity of each drive, which is a random number between seven tenths and eight tenths of the total drive capacity. Note that in the appendix, all energy values are in Joules and all time values are in seconds. Values are given to four decimal places.

\section{Appendix A.1. Storage Cost Generation}

The storage costs for the Standard commands data set and the File Server data set are dependant on how much free space is available on each disk before the optimisation takes place. A full table will not be provided as it is more efficient to describe the computations that took place. For a given drive, it is supposed that the drive is between $70 \%$ and $80 \%$ full and the actual percentage value is determined via the MATLAB function 'rand'. The amount of data WRITTEN to the drive for a given command is converted to $\mathrm{Gb}$ and this is then divided by the amount of free space in Gb on the drive. Given the size of the commands and the fact that drives have such large amounts of space, the number computed is thus very small and so to 
improve model performance and to reduce rounding errors, one is added to each value. The storage costs will change during model operation as when commands are written to the drives, the amount of free space available will decrease.

Appendix A.2. Standard Commands Data Set

Table A.10 and table A.11 contain the energy and time values for the Standard commands data set. That is, for each of the 24 commands and the 5 drive types used.

\section{Appendix A.3. File Server Data Set}

As stated, there are 100 commands in total and they are made up of the 8 different transfer sizes listed in tables A.12 and A.13. For reasons of space, the energy and time values for every command was not listed. The above tables provide a sample energy and time value for the different data transfer values and each drive. There is a slight variability so energy and time values for other command examples not listed will be slightly different. 
Table A.10: Measured Energy Values for each Drive Type on the Standard Command Data Set

\begin{tabular}{llllll}
\hline Profile Number & Drive 1 & Drive 2 & Drive 3 & Drive 4 & Drive 5 \\
\hline 1 & 0.0095 & 0.0238 & 0.0177 & 0.0379 & 0.0000 \\
\hline 2 & 0.0120 & 0.0262 & 0.0179 & 0.0451 & 0.0008 \\
\hline 3 & 0.0133 & 0.0248 & 0.0186 & 0.0410 & 0.0001 \\
\hline 4 & 0.0095 & 0.0003 & 0.0003 & 0.0001 & 0.0000 \\
\hline 5 & 0.0010 & 0.0025 & 0.0039 & 0.0051 & 0.0001 \\
\hline 6 & 0.0011 & 0.0037 & 0.0073 & 0.0069 & 0.0001 \\
\hline 7 & 0.0097 & 0.0236 & 0.0178 & 0.0389 & 0.0000 \\
\hline 8 & 0.0118 & 0.0251 & 0.0183 & 0.0460 & 0.0001 \\
\hline 9 & 0.0132 & 0.0244 & 0.0187 & 0.0407 & 0.0001 \\
\hline 10 & 0.0001 & 0.0002 & 0.0002 & 0.0001 & 0.0000 \\
\hline 11 & 0.0008 & 0.0021 & 0.0039 & 0.0032 & 0.0001 \\
\hline 12 & 0.0011 & 0.0033 & 0.0072 & 0.0034 & 0.0001 \\
\hline 13 & 0.0102 & 0.0234 & 0.0193 & 0.0395 & 0.0004 \\
\hline 14 & 0.0124 & 0.0265 & 0.0199 & 0.0457 & 0.0011 \\
\hline 15 & 0.0135 & 0.0255 & 0.0203 & 0.0397 & 0.0015 \\
\hline 16 & 0.0010 & 0.0011 & 0.0009 & 0.0010 & 0.0004 \\
\hline 17 & 0.0028 & 0.0041 & 0.0053 & 0.0040 & 0.0010 \\
\hline 18 & 0.0023 & 0.0041 & 0.0079 & 0.0014 & 0.0007 \\
\hline 19 & 0.0471 & 0.0729 & 0.0648 & 0.1159 & 0.0249 \\
\hline 20 & 0.0383 & 0.0566 & 0.0473 & 0.1078 & 0.0251 \\
\hline 21 & 0.0306 & 0.0383 & 0.0319 & 0.0678 & 0.0248 \\
\hline 24 & 0.0152 & 0.0178 & 0.0127 & 0.0168 & 0.0059 \\
\hline & 0.0247 & 0.0248 & 0.0211 & 0.0295 & 0.0140 \\
\hline 22 & 0.0208 & 0.0177 & 0.0188 & 0.0172 & 0.0101 \\
\hline 17 & & & & & \\
\hline 2 & &
\end{tabular}


Table A.11: Measured Time Values for each Drive Type on the 24 Commands Data Set

\begin{tabular}{|c|c|c|c|c|c|}
\hline Profile Number & Drive 1 & Drive 2 & Drive 3 & Drive 4 & Drive 5 \\
\hline 1 & 0.0069 & 0.0083 & 0.0081 & 0.0093 & 0.0003 \\
\hline 2 & 0.0079 & 0.0092 & 0.0082 & 0.0109 & 0.0003 \\
\hline 3 & 0.0080 & 0.0090 & 0.0085 & 0.0102 & 0.0004 \\
\hline 4 & 0.0002 & 0.0002 & 0.0003 & 0.0001 & 0.0002 \\
\hline 5 & 0.0035 & 0.0045 & 0.0033 & 0.0030 & 0.0003 \\
\hline 6 & 0.0044 & 0.0064 & 0.0064 & 0.0042 & 0.0004 \\
\hline 7 & 0.0072 & 0.0085 & 0.0081 & 0.0094 & 0.0003 \\
\hline 8 & 0.0078 & 0.0089 & 0.0084 & 0.0110 & 0.0003 \\
\hline 9 & 0.0079 & 0.0093 & 0.0086 & 0.0103 & 0.0004 \\
\hline 10 & 0.0002 & 0.0002 & 0.0001 & 0.0001 & 0.0002 \\
\hline 11 & 0.0025 & 0.0036 & 0.0033 & 0.0020 & 0.0003 \\
\hline 12 & 0.0044 & 0.0062 & 0.0064 & 0.0021 & 0.0004 \\
\hline 13 & 0.0077 & 0.0094 & 0.0085 & 0.0099 & 0.0006 \\
\hline 14 & 0.0091 & 0.0103 & 0.0096 & 0.0114 & 0.0008 \\
\hline 15 & 0.0097 & 0.0105 & 0.0099 & 0.0106 & 0.0007 \\
\hline 16 & 0.0007 & 0.0005 & 0.0004 & 0.0004 & 0.0003 \\
\hline 17 & 0.0045 & 0.0049 & 0.0041 & 0.0020 & 0.0006 \\
\hline 18 & 0.0050 & 0.0068 & 0.0067 & 0.0006 & 0.0006 \\
\hline 19 & 0.0350 & 0.0343 & 0.0251 & 0.0355 & 0.0074 \\
\hline 20 & 0.0267 & 0.0266 & 0.0200 & 0.0325 & 0.0065 \\
\hline 21 & 0.0196 & 0.0188 & 0.0154 & 0.0224 & 0.0056 \\
\hline 22 & 0.0104 & 0.0075 & 0.0055 & 0.0063 & 0.0024 \\
\hline 23 & 0.0174 & 0.0146 & 0.0109 & 0.0119 & 0.0038 \\
\hline 24 & 0.0148 & 0.0137 & 0.0118 & 0.0063 & 0.0036 \\
\hline
\end{tabular}


Table A.12: Measured Energy Values for each Drive Type on the File Server Data Set

\begin{tabular}{llllll}
\hline Data Transfer Size $[\mathrm{kB}]$ & Drive 1 & Drive 2 & Drive 3 & Drive 4 & Drive 5 \\
\hline 0.5 & 0.0155 & 0.0196 & 0.0184 & 0.0554 & 0.0000 \\
\hline 1 & 0.0138 & 0.0210 & 0.0181 & 0.0526 & 0.0001 \\
\hline 2 & 0.0141 & 0.0207 & 0.0179 & 0.0525 & 0.0001 \\
\hline 4 & 0.0122 & 0.0203 & 0.0176 & 0.0528 & 0.0000 \\
\hline 8 & 0.0111 & 0.0200 & 0.0176 & 0.0519 & 0.0002 \\
\hline 32 & 0.0110 & 0.0210 & 0.0181 & 0.0514 & 0.0001 \\
\hline 64 & 0.0111 & 0.0206 & 0.0184 & 0.0507 & 0.0001 \\
\hline
\end{tabular}

Table A.13: Measured Time Values for each Drive Type on the File Server Data Set

\begin{tabular}{llllll}
\hline Data Transfer Size $[\mathrm{kB}]$ & Drive 1 & Drive 2 & Drive 3 & Drive 4 & Drive 5 \\
\hline 0.5 & 0.0065 & 0.0093 & 0.0083 & 0.0141 & 0.0003 \\
\hline 1 & 0.0062 & 0.0105 & 0.0089 & 0.0133 & 0.0001 \\
\hline 2 & 0.0064 & 0.0104 & 0.0084 & 0.0134 & 0.0002 \\
\hline 4 & 0.0074 & 0.0101 & 0.0081 & 0.0132 & 0.0003 \\
\hline 8 & 0.0077 & 0.0099 & 0.0081 & 0.0131 & 0.0004 \\
\hline 32 & 0.0078 & 0.0106 & 0.0082 & 0.0129 & 0.0002 \\
\hline 64 & 0.0078 & 0.0103 & 0.0085 & 0.0127 & 0.0003 \\
\hline
\end{tabular}




\section{References}

1. Data centre efficiency assessment. 2014. URL http://on.nrdc.org/ $10 \mathrm{cXHmD}$.

2. Chou J, Kim J, Rotem D. Energy-aware scheduling in disk storage systems. In: Distributed Computing Systems (ICDCS), 2011 31st International Conference on. IEEE; 2011:423-433.

3. Benini L, Micheli Gd. System-level power optimization: techniques and tools. ACM Transactions on Design Automation of Electronic Systems (TODAES) 2000;5(2):115-192.

4. Bisson T, Brandt SA, Long DD. A hybrid disk-aware spin-down algorithm with i/o subsystem support. In: Performance, computing, and communications conference, 200\%. IPCCC 200\%. IEEE Internationa. IEEE; 2007:236-245.

5. Irani S, Singh G, Shukla SK, Gupta RK. An overview of the competitive and adversarial approaches to designing dynamic power management strategies. Very Large Scale Integration (VLSI) Systems, IEEE Transactions on 2005;13(12):1349-1361.

6. Gurumurthi S, Sivasubramaniam A, Kandemir M, Franke H. Reducing disk power consumption in servers with drpm. Computer 2003;36(12):59-66.

7. Carrera EV, Pinheiro E, Bianchini R. Conserving disk energy in network servers. In: Proceedings of the 17th annual international conference on Supercomputing. ACM; 2003:86-97. 
8. Papathanasiou AE, Scott ML. Energy efficient prefetching and caching. In: Proceedings of the 2004 USENIX Annual Technical Conference. 2004:255-268.

9. Narayanan D, Donnelly A, Rowstron A. Write off-loading: Practical power management for enterprise storage. ACM Transactions on Storage (TOS) 2008;4(3):10.

10. Verma A, Koller R, Useche L, Rangaswami R. Srcmap: Energy proportional storage using dynamic consolidation. In: FAST; vol. 10. 2010:267280.

11. Wildani A, Miller EL. Semantic data placement for power management in archival storage. In: Petascale Data Storage Workshop (PDSW), 2010 5th. IEEE; 2010:1-5.

12. Leverich J, Kozyrakis C. On the energy (in) efficiency of hadoop clusters. ACM SIGOPS Operating Systems Review 2010;44(1):61-65.

13. Härder T, Hudlet V, Ou Y, Schall D. Energy efficiency is not enough, energy proportionality is needed! In: Database Systems for Adanced Applications. Springer; 2011:226-239.

14. Drwal M. Algorithm for quadratic semi-assignment problem with partition size coefficients. Optimization Letters 2014;8(3):1183-1190.

15. Vasudevan M, Tian YC, Tang M, Kozan E. Profiling: an application assignment approach for green data centers. In: 40th Annual Conference of the IEEE Industrial Electronics Society (IECON 2014). IEEE; 2014:5400-5406. 
16. Jones D, Jimenez M. Incorporating additional meta-objectives into the extended lexicographic goal programming framework. European Journal of Operational Research 2013;227(2):343-349.

17. Oddoye J, Jones D, Tamiz M, Schmidt P. Combining simulation and goal programming for healthcare planning in a medical assessment unit. European Journal of Operational Research 2009;193(1):250 - 261. doi: \bibinfo\{doi\}\{http://dx.doi.org/10.1016/j.ejor.2007.10.029\}. URL http://bit.1y/1WFODty.

18. Ghoseiri K, Ghannadpour SF. Multi-objective vehicle routing problem with time windows using goal programming and genetic algorithm. Applied Soft Computing 2010;10(4):1096 - 1107. doi:\bibinfo\{doi\} \{http://dx.doi.org/10.1016/j.asoc.2010.04.001\}. Optimisation Methods \&amp; Applications in Decision-Making Processes; URL http://bit. ly/1 JMLLBK.

19. Tao F, Feng Y, Zhang L, Liao T. Clps-ga: A case library and pareto solution-based hybrid genetic algorithm for energy-aware cloud service scheduling. Applied Soft Computing 2014;19(0):264 - 279. doi:\bibinfo\{doi\}\{http://dx.doi.org/10.1016/j.asoc.2014.01.036\}. URL http://bit.1y/1Lr8ACu.

20. Guzek M, Pecero JE, Dorronsoro B, Bouvry P. Multi-objective evolutionary algorithms for energy-aware scheduling on distributed computing systems. Applied Soft Computing 2014;24(0):432 - 446. doi: \bibinfo\{doi\}\{http://dx.doi.org/10.1016/j.asoc.2014.07.010\}. URL http://bit.1y/1JMLP17. 
21. Operating systems and benchmarks - part 5. 2000. Accessed 5th September 2016; URL http://bit.1y/2cH35mx.

22. Ho SC. An iterated tabu search heuristic for the single source capacitated facility location problem. Applied Soft Computing 2015;27:169178.

23. Akpinar S, Bayhan GM, Baykasoglu A. Hybridizing ant colony optimization via genetic algorithm for mixed-model assembly line balancing problem with sequence dependent setup times between tasks. Applied Soft Computing 2013;13(1):574-589. 